\title{
A Method to Correct for Brain Shift When Building Electrophysiological Atlases for Deep Brain Stimulation (DBS) Surgery
}

\author{
Srivatsan Pallavaram ${ }^{1}$, Benoit M. Dawant ${ }^{1}$, Rui $\mathrm{Li}^{1}$, Joseph S. Neimat ${ }^{2}$, \\ Michael S. Remple ${ }^{2}$, Chris $\mathrm{Kao}^{2}$, Peter E. Konrad ${ }^{2}$, and Pierre-François D’Haese ${ }^{1}$ \\ ${ }^{1}$ Dept. of Electrical Engineering and Computer Science \\ ${ }^{2}$ Dept. of Neurosurgery, \\ Vanderbilt University, Nashville, TN, USA
}

\begin{abstract}
To help surgeons to pre-operatively select the target location for DBS electrodes, functional atlases based on intra-operatively acquired data have been created in the past. Recently, many groups have reported on the occurrence of brain shift in stereotactic surgery and its impact on the procedure but not on the creation of such atlases. Due to brain shift, the pre- and intra-operative coordinates of anatomic structures are different. When building large population atlases, which rely on pre-operative images for normalization purposes, it is thus necessary to correct for this difference. In this paper, we propose a method to achieve this. We show evidence that electrophysiological maps built using corrected and uncorrected data are different and that the maps created using shiftcorrected data correlate better than those created using uncorrected data with the final position of the implant. These findings suggest that brain-shift correction of intra-operatively recorded data is feasible for the construction of accurate shift-corrected electrophysiological atlases.
\end{abstract}

\section{Introduction}

Deep brain stimulation (DBS) is a surgical procedure involving the implantation of an electrode in the deep brain to stimulate specific nuclei using a pacemaker. DBS has provided remarkable therapeutic benefits to patients suffering from movement disorders such as the Parkinson's disease. To help the surgeon pre-operatively select the target location for the electrode, functional atlases $[1,2]$ based on intra-operatively acquired electrophysiological data from a number of patients have been created to complement anatomical and histological atlases. Several authors [1,3-5] have shown techniques by which such atlases can be used in the planning, placement and programming of DBS.

An underlying assumption in the creation of these atlases is that anatomical structures do not move between pre-operative imaging and intra-operative recording. A number of studies have proved that this assumption is not valid. Miyagi et al. [6] found the anterior and posterior commissures to be more medial, posterior and inferior on the post-operative MRI than on the pre-operative MRI. Khan et al. [7] reported brain shifts of up to $4 \mathrm{~mm}$ in deep brain structures. Using real-time intraoperative MRI Martin et al. [8] recently reported appreciable ipsilateral brain shift 
during burr hole access. Consequently, the pre- and intra-operative coordinates of anatomic structures may be different. But, to create population atlases, pre-operative image volumes are typically utilized because the stereotactic platform that is used to reference the intra-operative data is built in the pre-operative image space. It is thus critical to correct for brain shift to place data recorded intra-operatively at the correct location in the pre-operative scans. In this paper, we propose a method to do this. We also present preliminary results which indicate that shift correction has a substantial effect on statistical maps derived from these data and that shift-corrected maps are more accurate than maps computed with uncorrected data.

\section{Data}

With IRB approval each patient had pre-operative MRI and CT, a post-operative CT acquired on the day of the surgery, and a post-operative stable CT acquired approximately one month after surgery before programming of the electrode. The scan acquired the day of surgery will be called post-op CT, the scan acquired one month after the procedure will be called stable CT. Typical CT images were acquired at $\mathrm{kVp}=$ $120 \mathrm{~V}$, exposure $=350 \mathrm{mAs}$ and $512 \times 512$ pixels. In-plane resolution and slice thickness were respectively $0.5 \mathrm{~mm}$ and $0.75 \mathrm{~mm}$. MRI (TR $12.2 \mathrm{~ms}$, TE $2.4 \mathrm{~ms}$, $256 \times 256 \times 170$ voxels, with typical voxel resolution of $1 \times 1 \times 1 \mathrm{~mm}^{3}$ ) were acquired using the SENSE parallel imaging technique (T1W/3D/TFE) from Philips on a $3 T$ scanner. We used patients that underwent sub-thalamic nucleus (STN) targeting. Stimulation data included the location of each stimulation point, the efficacy (therapeutic response) observed and the associated stimulation current, the adverse effect (if any) and the associated current. Efficacy was recorded as percentage reduction in symptoms from baseline as assessed by a neurologist. Only those points with at least $70 \%$ efficacy were used. The dataset comprised of 36 efficacious points and 35 eye deviation points (an adverse effect) from 13 patients for the left side, 20 efficacious points from 8 patients and 34 eye deviation points from 12 patients for the right side. Some other adverse effects include muscular contraction, dysarthria, or parasthesia. We have chosen to use eye deviation data in this work because it is the most populous adverse effect in our dataset.

\section{Method}

To create atlases, the 3D T1-weighted images were registered to each other using a combination of intensity-based rigid and non-rigid registration algorithms. The nonrigid registration we proposed earlier [9] is called the Adaptive Bases Algorithm (ABA). Briefly, it computes a deformation field that is modeled as a linear combination of radial basis functions with finite support. This results in a transformation with several thousands of degrees of freedom. Two transformations (one from the atlas to the subject and the other from the subject to the atlas) that are constrained to be inverses of each other are computed simultaneously. ABA reduces the computational complexity and improves the convergence properties of related B-splinesbased approaches by identifying regions of mis-registration and adapting the 
compliance of the transformation locally. The algorithm arrives at the final deformation iteratively across scales and resolutions and uses mutual information as the similarity measure.

One solution to build shift-free functional atlases is to use only those patients that have minimum or no intra-operative shift (based on pneumocephalus in immediate CT) as shown by us in [10]. Patients were placed in the low, medium and large shift category based on the average air pocket width (AAPW) at the cortical surface seen on the post-op CT. To determine the region in the frontal cortex where cortical surface shift was most likely to cause shift at the target, a reference line parallel to the direction of gravity and passing through the implant was drawn and average of 4 air width measurements made in its vicinity was computed. Using data from patients in the low category (AAPW $\leq 3 \mathrm{~mm}$ ) we populated shift-free atlases. These shift-free atlases are built without any correction applied to the intra-op coordinates. The limitation of this approach is that a large number of patients' data (medium: $3 \mathrm{~mm}<$ AAPW $\leq 7 \mathrm{~mm}$ and large group: AAPW $>7 \mathrm{~mm}$ ) cannot be used.

The shift correction method we propose permits using all patients to build statistical maps. Fig 1(a) shows a detailed model of various shift components.

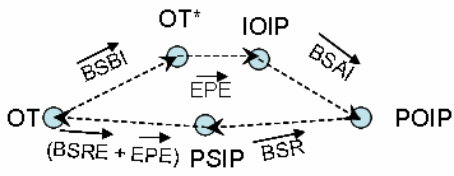

(a) Various brain shift components and stages

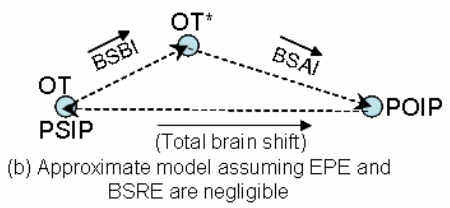

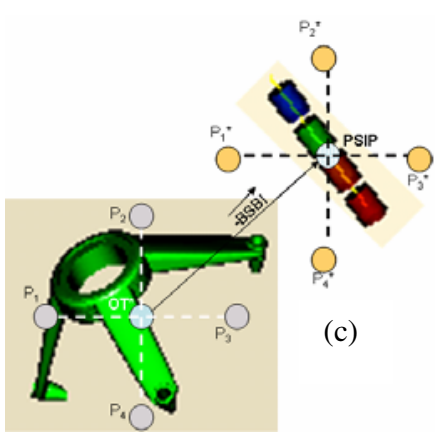

Fig. 1. (a) Detailed model of various brain shift components, (b) approximate model, (c) demonstrating how data points $\mathrm{P}_{1}, \mathrm{P}_{2}, \mathrm{P}_{3}$ and $\mathrm{P}_{4}$ are corrected for brain shift to arrive at $\mathrm{P}_{1}{ }^{*}, \mathrm{P}_{2}{ }^{*}, \mathrm{P}_{3}{ }^{*}$, $\mathrm{P}_{4}{ }^{*}$ using the model in (b). OT: optimal target, $(\overrightarrow{B S B I})$ : Brain Shift Before Implantation, OT* shifted position of OT due to $(\overrightarrow{B S B I})$ and identified as the location for implantation by electrophysiological mapping, $(\overrightarrow{E P E})$ : Electrode Placement Error, IOIP: Intra-Operative Implant Position, $(\overrightarrow{B S A I})$ : Brain Shift After Implantation, POIP: Post-Operative Implant Position as seen on the CT acquired immediately after surgery, $(\overrightarrow{B S R})$ : Brain Shift Recovery, PSIP: Postoperative Stable Implant Position as seen on the stable CT acquired about a month after surgery, $(\overrightarrow{B S R E})$ : Brain Shift Recovery Error.

During the procedure, the optimal target (OT) where the implant should ideally be placed gets displaced due to Brain Shift Before Implantation $(\overrightarrow{B S B I})$ and moves to $\mathrm{OT}^{*}$. By electrophysiological mapping $\mathrm{OT}^{*}$ is identified as the location for implantation. Electrode Placement Error $(\overrightarrow{E P E})$ due to the finite accuracy of the stereotactic 
system and/or manual errors causes the electrode to be implanted at a slightly different location. This is the Intra-Operative Implant Position (IOIP). Brain Shift After Implantation $(\overrightarrow{B S A I})$ causes the implant to move further to the Post-Operative Implant Position (POIP) as seen on the post-op CT. Finally, due to Brain Shift Recovery $(\overrightarrow{B S R})$ the lead stabilizes at the Post-operative Stable Implant Position (PSIP) by the time the stable CT is acquired. The difference between PSIP and OT is because of $(\overrightarrow{E P E})$ and/or Brain Shift Recovery Error $(\overrightarrow{B S R E})$ due to the brain not recovering exactly to its pre-operative state. If we assume that $(\overrightarrow{B S R E})$ and $(\overrightarrow{E P E})$ are negligible, the model reduces to the simpler formulation shown in fig 1(b) where PSIP returns to OT. Using this model we can account for brain shift when populating atlases. The intra-operative coordinates of all the data points $\left(\mathrm{P}_{1}, \mathrm{P}_{2}, \mathrm{P}_{3}\right.$ and $\mathrm{P}_{4}$ in fig 1(c)) and OT are known in the platform coordinates. Probabilistic maps built using these coordinates are referred to as uncorrected maps. Using the approximate model, the transformation between $\mathrm{OT}^{*}$ and PSIP is $-\overrightarrow{B S B I}$. By applying this transformation to all intra-operative points, their corrected coordinates $\left(\mathrm{P}_{1}{ }^{*}, \mathrm{P}_{2}{ }^{*}, \mathrm{P}_{3}{ }^{*}, \mathrm{P}_{4}{ }^{*}\right)$ can be computed as illustrated in fig 1(c). By populating electrophysiological atlases using these coordinates we can build shift-corrected atlases and maps referred to as corrected maps. Probabilistic maps were created from atlases of intra-operative measurements using the method proposed by us in [3].

\section{Results}

Our method is based on the assumption that, in the stable CT, brain has recovered to its pre-operative state. This assumption is difficult to prove. However, if it is correct, the lead position in the stable CT should correspond to a region of high efficacy predicted by our shift-free map. To verify this hypothesis we have used two patients in which we observed substantial shift $(3.14 \mathrm{~mm}$ and $2.90 \mathrm{~mm}$ at the target position). Fig 2 illustrates our results. In both patients, we show the location identified intraoperative as the optimal target for implantation (OT ${ }^{*}$ ) shown by 4 dashed contacts and the stable lead position (solid contacts) overlaid on shift-free efficacy maps. Since shift-free maps are the closest to ground truth i.e. maps unaffected by shift, and because as per the approximate mode the stable lead position (PSIP) is the shiftrecovered position of the implant $\left(\mathrm{OT}^{*}\right)$, these results show that our assumption of negligible brain shift recovery is reasonable because PSIP correlates better than OT with shift-free maps. To further test our assumption, we would ideally compare shiftfree with corrected and uncorrected efficacy maps. Due to the relatively small number of shift-free cases for which we have stable CTs a quantitative comparison is not possible at the current time. To address this issue we validate our approach in two steps: (1) we show that corrected and uncorrected maps are different, and (2) we show that the position of the electrode in the stable CT correlates better with zones of high efficacy predicted by the shift-corrected maps than with those predicted by uncorrected maps. 


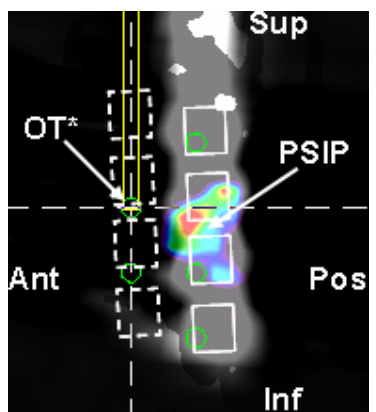

(a)

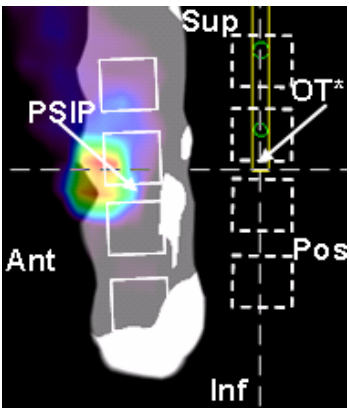

(b)

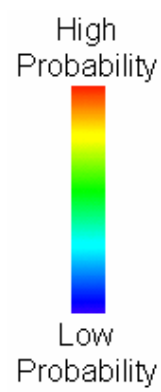

(c)

Fig. 2. Sagittal view showing relative positions of the intra-operative optimal target $\left(\mathrm{OT}^{*}\right)$ as dotted contacts, actual lead from the stable CT (PSIP) as solid contacts overlaid on shift-free maps built for two patients (a) Patient1, (b) Patient2. (c) Color scale. The gray region extending out of the core of the map and masking the stable lead is the low probability region of the map.

To show that the maps are different, we first compute the Dice similarity coefficient (S) [11] between two sets defined in (1), (2), and (3) where $p(\mathrm{X}, \mathrm{Y}, \mathrm{Z})$ is the probability value at point $(\mathrm{X}, \mathrm{Y}, \mathrm{Z})$ in a map.

$$
\begin{gathered}
\Omega_{\text {uncorrected }}=\left\{(X, Y, Z), \ni p_{\text {uncorrected }}(X, Y, Z) \geq \text { threshold }\right\} \\
\Omega_{\text {corrected }}=\left\{(X, Y, Z), \ni p_{\text {corrected }}(X, Y, Z) \geq \text { threshold }\right\} \\
S=\frac{2 * N\left(\left\{\Omega_{\text {uncorrected }} \cap \Omega_{\text {corrected }}\right\}\right)}{N\left(\Omega_{\text {uncorrected }}\right)+N\left(\Omega_{\text {corrected }}\right)} \\
N(\Omega) \text { is the number of elements in any set } \Omega
\end{gathered}
$$

We then compare connectivity and spread of the maps. Because physiological regions should concentrate into well connected tight clusters, we compare the maps by computing the number of clusters (NOC) with high probability. We do this using 3dimensional 26-connectivity where voxels sharing any of their 6 faces, 12 edges or 8 corners are grouped into the same cluster. A larger number of such clusters indicates greater number of isolated high probability zones and poor overall clustering. To measure the spread of the maps, we computed the mean distance from cluster centroid (MDCC) for points of high probability. Table 1(a) gives the values of dice coefficient for the uncorrected and corrected maps of efficacy (EFF) and eye deviation (EYE). Table 1(b) shows the number of 26-connected clusters in the two maps for high probability points and the mean distance from cluster centroid for those points. This table shows that the uncorrected and corrected maps are very different. From table 1(a), the uncorrected and corrected efficacy and eye deviation maps for the the left and right sides had respectively $57 \%, 44 \%, 57 \%$ and $46 \%$ of non-zero probability voxels overlapping. Considering only voxels with high probability $(\mathrm{p}>=0.7)$, the percentage of overlapping voxels decreased to $0 \%, 12 \%, 0 \%$ and $8 \%$ respectively indicating that the high probability regions in the two maps were almost completely different. Table 1(b) shows that the number of distinct high probability clusters is generally higher in the uncorrected maps than in the corrected maps; 4 to 1 for left efficacy, 8 to 1 for right 
eye deviation and 2 to 1 for left eye deviation. This indicates that clustering tends to be tighter in the corrected maps than in the uncorrected maps. In turn, this suggests that corrected maps represent electrophysiology better than uncorrected maps. This is further supported by the larger mean distances from cluster centroid for uncorrected maps than for corrected maps. Fig 3 and fig 4 show coronal, axial and sagittal slices of (a) corrected and (b) uncorrected maps overlaid over the stable CT for the two large-shift patients used earlier. Since we showed in fig 2 that shift recovery error can be neglected, it means that the stable lead position or PSIP returns to the optimal target location in a patient. Therefore, if shift-corrected maps built for a patient were correct then they would correlate better than uncorrected maps with PSIP. This can be seen in fig 3 and fig 4.

Table 1. (a) Dice coefficient (S) for uncorrected (uncorr) and corrected (corrr) maps, EFF: efficacy, EYE: eye deviation, (b) Number of clusters (NOC) in the two maps for $p>=0.7$ and 26-connectivity, and, mean distance from cluster centroid (MDCC) for $\mathrm{p}>=0.7$

\begin{tabular}{|c|c|c|}
\hline \multirow{2}{*}{ (a) } & \multicolumn{2}{|c|}{$\mathbf{S}$} \\
\cline { 2 - 3 } & $\mathbf{p}>\mathbf{0}$ & $\mathbf{p}>\mathbf{= 0 . 7}$ \\
\hline EFF LEFT & 0.57 & 0 \\
\hline EFF RIGHT & 0.44 & 0.12 \\
\hline EYE LEFT & 0.57 & 0 \\
\hline EYE RIGHT & 0.46 & 0.08 \\
\hline
\end{tabular}

\begin{tabular}{|c|c|c|c|}
\hline (b) & Map & NOC & MDCC \\
\hline \multirow{2}{*}{ EFF LEFT } & Corr & 1 & 1.29 \\
\cline { 2 - 4 } & Uncorr & 4 & 3.25 \\
\hline \multirow{2}{*}{ EFF RIGHT } & Corr & 2 & 2.5 \\
\cline { 2 - 4 } & Uncorr & 2 & 2.8 \\
\hline \multirow{2}{*}{ EYE LEFT } & Corr & 1 & 1.27 \\
\cline { 2 - 4 } & Uncorr & 2 & 1.67 \\
\hline \multirow{2}{*}{ EYE RIGHT } & Corr & 1 & 1.9 \\
\cline { 2 - 4 } & Uncorr & 8 & 3.64 \\
\hline
\end{tabular}

(a)
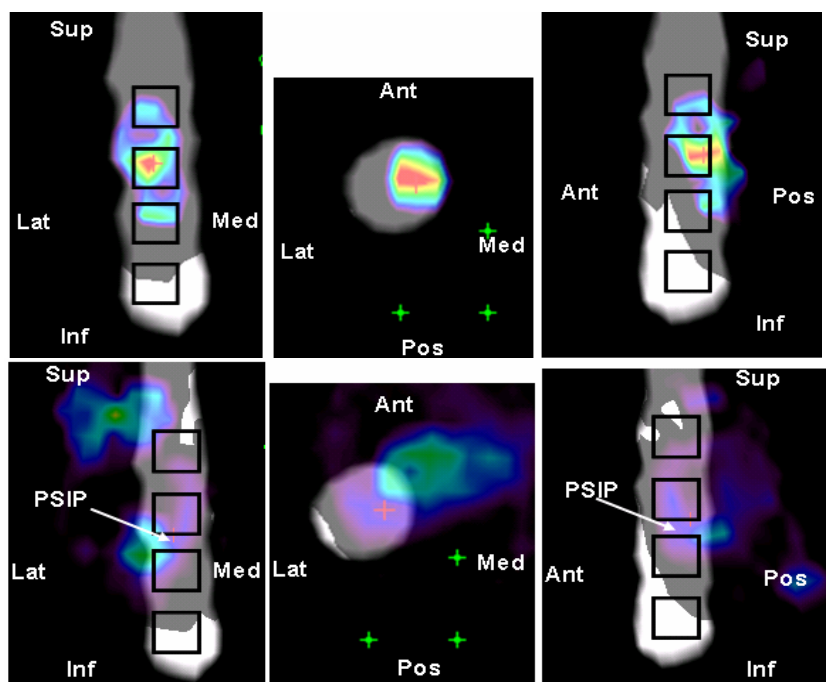

Fig. 3. Coronal, axial and sagittal slices of (a) corrected and (b) uncorrected maps overlaid over the stable CT for patient1. The color scale is shown in fig 2(c). 
(a)
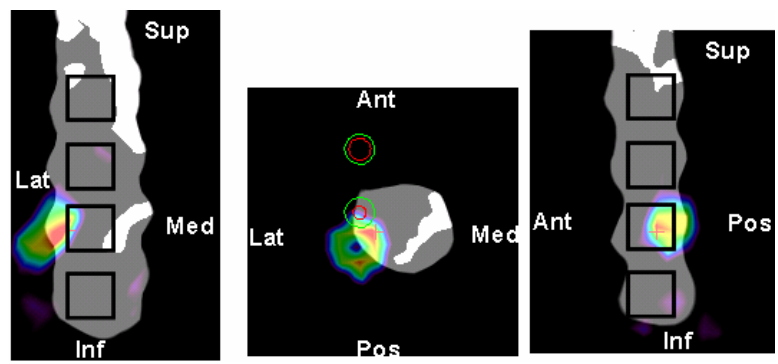

(b)
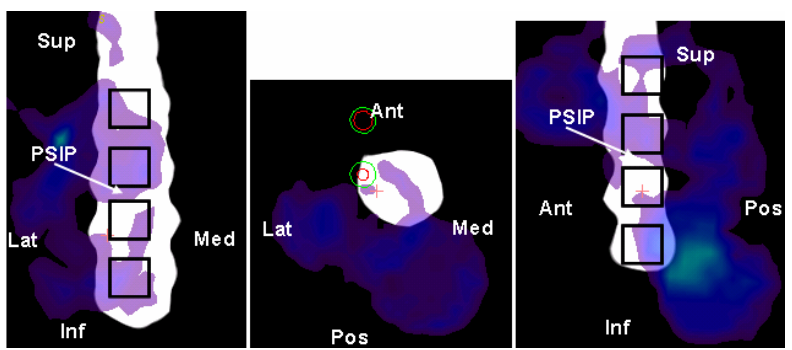

Fig. 4. Coronal, axial and sagittal slices of (a) corrected and (b) uncorrected maps overlaid over the stable CT for patient2. Color scale is shown in fig 2(c)

\section{Discussion}

Creation of accurate efficacy and side effect maps is important for the pre-operative planning and intra-operative guidance of DBS procedures. Stimulation data acquired during the procedures is a rich source of information to build these maps, but substantial brain shift (up to $4 \mathrm{~mm}$ ) in areas surrounding the regions of implantation has been reported in the literature. Because of this shift, the intra-operative coordinates of a structure may be different from its pre-operative coordinates. In turn, this affects the accuracy of maps derived from these data. It has been reported that shift is related to the amount of air entering the cranial cavity during the procedure. A straightforward approach to building the maps would thus be to screen patients and keep only those for whom there is minimum air invasion. While feasible, this approach also severely limits the number of data sets which can be used to create statistical maps. In this paper, we present an approach that permits the correction of this shift and thus the use of data that would otherwise need to be discarded. Albeit preliminary, the results we present strongly suggest that our approach produces efficacy maps that correlate better with the anatomical location selected as optimal during the procedure than the uncorrected maps. This optimal anatomical target location is referred to as OT, which according to our approximate model is the stable lead position (PSIP). Work is ongoing to validate these results in a large number of patients and to correlate high efficacy zones predicted using shift-corrected maps of intra-operative data with post-operative programming stimulation response observations.

Acknowledgment. This research has been supported, in parts, by NIH R01 EB006136. 


\section{References}

1. D'Haese, P.-F., Cetinkaya, E., Konrad, P.E., Kao, C., Dawant, B.M.: Computer-Aided Placement of Deep Brain Stimulators: From Planning to Intraoperative Guidance. IEEE Trans. on Medical Imaging. 24(11), 1469-1478 (2005)

2. Finnis, K.W., Starreveld, Y.P., Parrent, A.G., Sadikot, A.F., Peters, T.M.: Three dimensional database of subcortical electrophysiology for mage-guided stereotactic functional neurosurgery. IEEE Trans. on Medical Imaging 22(11), 93-104 (2003)

3. Pallavaram, S., D’Haese, P.-F., Kao, C., Yu, H., Remple, M., Neimat, J.S., Konrad, P.E., Dawant, B.M.: A new method for creating electrophysiological maps for DBS surgery and their application to surgical guidance. In: Metaxas, D., Axel, L., Fichtinger, G., Székely, G. (eds.) MICCAI 2008, Part I. LNCS, vol. 5241, pp. 670-677. Springer, Heidelberg (2008)

4. D’Haese, P.-F., Pallavaram, S., Yu, H., Spooner, J., Konrad, P.E., Dawant, B.M.: Deformable Physiological Atlas-Based Programming of Deep Brain Stimulators: A Feasibility Study. In: Pluim, J.P.W., Likar, B., Gerritsen, F.A. (eds.) WBIR 2006. LNCS, vol. 4057, pp. 144-150. Springer, Heidelberg (2006)

5. Guo, T., Finnis, K.W., Parrent, A.G., Peters, T.M.: Development and application of functional databases for planning deep-brain neurosurgical procedures. In: Duncan, J.S., Gerig, G. (eds.) MICCAI 2005. Part I. LNCS, vol. 3749, pp. 835-842. Springer, Heidelberg (2005)

6. Miyagi, Y., Shima, F., Sasaki, T.: Brain shift: an error factor during implantation of deep brain stimulation electrodes. Neurosurgery 107, 989-997 (2007)

7. Khan, M.F., Mewes, K., Gross, R.E., Škrinjar, O.: Assessment of Brain Shift Related to Deep Brain Stimulation Surgery. Stereotact Funct Neurosurgery 86, 44-53 (2008)

8. Martin, A.J., Larson, P.S., Ostrem, J.L., Sootsman, W., Talke, P., Weber, O.M., Levesque, N., Myers, J., Starr, P.A.: Placement of deep brain stimulator electrodes using real-time highfield interventional magnetic resonance imaging. Magn. Reson. Med. 54, 1107-1114 (2005)

9. Rhode, G.K., Aldroubi, A., Dawant, B.M.: The adaptive bases algorithm for intensitybased nonrigid image registration. IEEE Trans. On Medical Imaging 22(11), 1470-1479 (2003)

10. Pallavaram, S., Remple, M., Neimat, J.S., Kao, C., Konrad, P.E., Dawant, B.M., D'Haese, P.-F.: Effect of brain shift on the creation of functional atlases for deep brain stimulation surgery. In: Computer Assisted Radiology and Surgery, Germany (2009)

11. Dice, L.R.: Measures of the amount of ecologic association between species. Ecology 26, 297-302 (1945) 